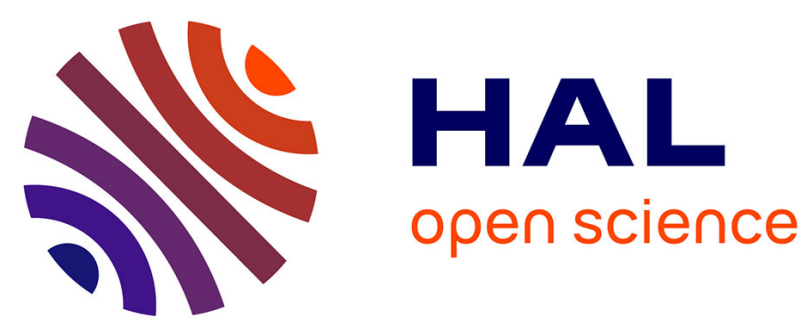

\title{
Blood-Brain Barrier Proteomics: Towards the Understanding of Neurodegenerative Diseases
}

Yannis Karamanos, Fabien Gosselet, Marie-Pierre Dehouck, Roméo Cecchelli

\section{To cite this version:}

Yannis Karamanos, Fabien Gosselet, Marie-Pierre Dehouck, Roméo Cecchelli. Blood-Brain Barrier Proteomics: Towards the Understanding of Neurodegenerative Diseases. Archives of Medical Research, 2014, 45 (8), pp.730-737. 10.1016/j.arcmed.2014.11.008 . hal-02515225

\section{HAL Id: hal-02515225 \\ https://hal-univ-artois.archives-ouvertes.fr/hal-02515225}

Submitted on 9 Dec 2021

HAL is a multi-disciplinary open access archive for the deposit and dissemination of scientific research documents, whether they are published or not. The documents may come from teaching and research institutions in France or abroad, or from public or private research centers.
L'archive ouverte pluridisciplinaire HAL, est destinée au dépôt et à la diffusion de documents scientifiques de niveau recherche, publiés ou non, émanant des établissements d'enseignement et de recherche français ou étrangers, des laboratoires publics ou privés.

\section{(ㅇ)(1) $\$$}

Distributed under a Creative Commons Attribution - NonCommerciall 4.0 International 


\title{
Archives \\ of Medical \\ Research
}

Archives of Medical Research — (2014) a

REVIEW ARTICLE

\section{Blood-Brain Barrier Proteomics: Towards the Understanding of Neurodegenerative Diseases}

\author{
Yannis Karamanos, Fabien Gosselet, Marie-Pierre Dehouck, and Roméo Cecchelli \\ Laboratoire de la Barrière Hématoencéphalique, Lens, France
}

Received for publication November 10, 2014; accepted November 12, 2014 (ARCMED-D-14-00649).

The blood-brain barrier (BBB) regulates the passage of endogenous and exogenous compounds and thus contributes to the brain homeostasis with the help of well-known proteins such as tight junction proteins, plasma membrane transporters and metabolic barrier proteins. In the last decade, proteomics have emerged as supplementary tools for $\mathrm{BBB}$ research. The development of proteomic technologies has provided several means to extend knowledge on the $\mathrm{BBB}$ and to investigate additional routes for the bypass of this barrier. Proteomics approaches have been used in vivo and also using in vitro $\mathrm{BBB}$ models to decipher the physiological characteristics and, under stress conditions, to understand the molecular mechanisms of brain diseases. This work has demonstrated that both quantitative global and targeted proteomics approaches are powerful and provide significant information on the brain microvessel endothelium. However, current knowledge is only partial and it is necessary to increase the studies using proteomics tools that will provide additional information concerning brain pathologies or BBB metabolism. Highly sensitive, accurate and specific protein quantification by quantitative targeted proteomics appears as an essential methodology for human BBB studies. (C) 2014 IMSS. Published by Elsevier Inc.

Key Words: Blood-brain barrier, BBB, Endothelial cells, Proteomics, Neurodegenerative disease. 
passage of signalling molecules across the BBB and (iv) cell signalling via the expression of PM receptors (10). Since its discovery 100 years ago, the BBB has been the center of thorough studies; however, it is only in more recent years that "omics" analyses have been used for its understanding.

\section{Proteomics Approaches for BBB}

The survey of the genomic and proteomic investigations carried out in order to enhance our understanding of the BBB (11) has demonstrated that expression profiling technologies are powerful tools and provide significant information on the brain microvessel endothelium. Certainly genomics and proteomics provide different data on a tissue but they are complementary; genomics underline relative expression of membrane and released proteins, whereas proteomics indicate protein modifications and isoform expression. Moreover, characterization of endothelial cells highlights some brain specific proteins, which could explain BBB features. Pioneer studies have identified some of the unique molecular characteristics of the vascular bed and have reinforced the concept that the BBB is heavily involved in brain function (12). Genomic and proteomic techniques have also been used to analyze the molecular events underlying diseases that have BBB involvement such as multiple sclerosis, Alzheimer's disease, stroke and HIV-1 infection. A comparative proteomic analysis of rat brain microvascular endothelial cells and coronary microvascular endothelial cells highlights the characteristic proteins of the BBB (13). Due to their specificity and their singular structures, the BEC have a particularity in their metabolic and structural proteins. Indeed, it has been shown (13) that cytoskeleton-associated proteins are involved in BEC differentiation, for example, the macrophage-capping protein $\mathrm{G}$, a gelsolin/villin family protein, interacts with actin to reduce actin filaments and cap end-barbed actin filaments. The interaction of this protein with the cytoskeleton and DNA may play a role in the regulation of the cytoplasmic and nuclear structure of actin. Moreover, in the aortic endothelial cells, this protein contributes to the increase in blood flow (14). In the same study, the chloride intracellular channel protein 4, the cytosol aminopeptidase, the protein calcium/calmodulin-dependent serine protein kinase, a membrane-associated guanylate kinase, and IL-6 were shown to be upregulated in the BEC.

Inderstanding of the functional roles of proteins in drug absorption, distribution, metabolism, elimination, toxicity, and efficacy (ADMET/efficacy) is important for drug discovery and development but, for this, detailed information about protein expression is required. The protein quantification method, called quantitative targeted absolute proteomics, has been developed on the basis of separation and identification of protein digests by liquid chromatography (LC)-linked tandem mass spectrometry (MS) with multiple reaction monitoring. Target peptides for quantification are selected only from sequence information, so timeconsuming procedures such as antibody preparation and protein purification are unnecessary. The high selectivity of quantitative targeted absolute proteomics and its ability to quantify multiple proteins simultaneously make it possible to determine the absolute expression levels of many proteins in tissues and cells in both physiological and disease states. Knowledge of absolute expression amounts, together with data on intrinsic protein activity, allows reconstruction of in vivo protein function; thus, this is an efficient strategy to predict ADMET/efficacy of drug candidates in humans in various disease states (15).

Both global and targeted proteomics strategies can be applied. Targeted proteomics strategies limit the number of features that will be monitored and then optimize the methods to obtain the highest sensitivity and throughput for a huge amount of samples. The advantage of global proteomics strategies is that no hypothesis is required other than a measurable difference in one or more protein species among the samples. Global proteomics methods attempt to separate, quantify, and identify all proteins from a given sample (16) and have the potential to clarify the unique attributes of a healthy BBB, to identify therapeutic targets in diseased brain, and to identify novel approaches for noninvasive delivery of drugs against these targets (17). The need for adapting existing proteomics techniques to identify and quantify transporter proteins at the PM was foreseen several years ago (18). In the rapidly evolving pharmacoproteomics area, Terasaki and co-workers opened a new field to reveal changes in BBB in disease, aging and assessment of individual differences and evaluate in vivo-in vitro differences (19).

Recent advances in both microdissection techniques and proteomic analytical tools allowed isolating relatively pure cell populations from complex tissues in situ and profiling of cellular proteomes. For example, immunohistochemistry-guided laser capture microdissection provides the unique opportunity to selectively investigate BEC from the surrounding cell populations at the $\mathrm{BBB}$, while supporting downstream proteomic analysis. Using such an approach to obtain comprehensive protein expression profiles of the cerebral endothelium in situ will enable detailed understanding of the crucial mediators of signaling and BBB function in both normal and pathophysiological conditions (20).

\section{In-gel Profiling}

A differential proteomic approach was initiated using the in vitro $\mathrm{BBB}$ model developed in the authors' laboratory and made up of pure BEC co-cultured with glial cells (21). Co-culture conditions induce differentiation of endothelial cells and characteristics, which resemble in vivo BBB phenotype (22). It was mandatory to adapt the 
technique to the model and to optimize the protocol to this culture mode. In preliminary experiments the particularities of the system were taken into account: (i) fragility of the cells, (ii) presence of the collagen matrix and (iii) presence of serum proteins in the medium. Because cell harvesting, cell lysis and protein extraction procedures are unavoidable inseparable components of any cellular proteomics approach, the reproducibility directly depends on the care taken to prepare the protein samples, and this could affect all subsequent steps and, of course, the final results.

The in-gel profiling approach helped to determine the changes occurring in BEC differentiated in co-culture with astrocytes compared with endothelial cells cultured alone. The two-dimensional gel electrophoresis (2-DE) steps, including the isoelectric focusing and SDS-PAGE conditions, the gel staining, the image acquisition and the comparative study were detailed elsewhere (23) together with examples of protein identification by peptide mass fingerprint measured by MALDI-TOF-MS and complementary data issued from peptide fragmentation fingerprints that allow successful protein identifications. Peptide mass fingerprint and peptide fragmentation fingerprints analyses provide complementary datasets and thus, more comprehensive sequence coverage of the BEC proteome, especially when they are combined. Preliminary work (21) demonstrated the role of actin-binding and -bundling proteins such as gelsolin, filamin-A, T-plastin and actin itself in the establishment of the in vitro BBB phenotype of $\mathrm{BEC}$ in response to stimulation by glial cells. We also speculated that $\mathrm{Ca}^{2+}$ and phosphatidylinositol-(3,4)-bis phosphate are involved in the response to this stimulation.

A consecutive thorough study (24) revealed differences in Triton-X-100-solubilized proteins from bovine BEC with limited or reinduced BBB functionalities (cultured in the absence or presence of glial cells, respectively). The 81 proteins of differing abundance were linked to 55 distinct genes. According to the PANTHER classification system and Ingenuity Pathway Analysis, these quantitative changes mainly affected proteins involved in (i) cell structure and motility and (ii) protein metabolism and modification processes. The fold-changes affecting heat-shock $27-\mathrm{kDa}$ protein 1, moesin and annexin A5 protein levels were confirmed by Western blot analysis but were not accompanied by changes in the corresponding mRNA expression levels. The results revealed that the BEC can adapt to variations in their environment and this involves the reorganization of the actin cytoskeleton contributing to the BBB phenotype.

A differential gel electrophoresis (2D-DIGE)-based proteomics approach (25) confirmed that quantitative changes mainly concern proteins involved in cell structure and motility. Furthermore, the possible involvement of the asymmetric dimethylarginine pathway in the BBB phenotype reinduction process was suggested and the potential role of asymmetric dimethylarginine in regulating endothelial function (in addition to its role as a by-product of protein modification) also foreseen. Purine nucleoside phosphorylase was also found to be more abundant in BEC with reinduced BBB phenotype. These results also suggested that the intracellular redox potential is lower in the in vitro brain capillary endothelial cells displaying reinduced BBB functions than in cells with limited BBB functions.

\section{Off-gel Profiling}

In a label-free off-gel profiling approach, the proteins in a sample are directly submitted to enzymatic digestion and the mixture of the generated peptides whose molecular mass ranges from 500-4,000 Da are separated and subsequently analyzed by MS. A differential nano-LC MALDI-TOF/TOF-MS study was performed with Triton $\mathrm{X}$-100-soluble protein species from bovine BEC displaying either limited or reinduced BBB functions (26). The complexity of the crude extract of Triton X-100 solubilized proteins from BEC was decreased by differential extraction into five fractions of increasing concentration in acetonitrile $(0,25,50,75$ and $100 \%)$ as previously described (27). Each fraction was subjected to the off-line 1D-LC separation in which high numbers of components were MS-detected; $\sim 15 \%$ were in-source fragmented and most of the MS/MS spectra provided unambiguous protein identities in the main fractions (F0, F25, F50 and F75). Overall, the analysis enabled the identification of 436 and 408 proteins in bovine BEC with limited and reinduced BBB, respectively. Eleven of these, ranging from proteins associated with assembly and organization of the cytoskeleton (21) to those involved in vesicular transport and nucleic acid binding, appeared to be more abundant in the cytoplasm of cells with reinduced BBB functions, in particular tissue nonspecific alkaline phosphatase and Eps15 homology domain-containing protein 1 . This overexpression was accompanied by an increase in alkaline phosphatase intracellular enzymatic activity. Moreover, we found that endothelial permeability was significantly greater when alkaline phosphatase activity was specifically inhibited with levamisole, suggesting that tissue nonspecific alkaline phosphatase is involved in the regulation of endothelial permeability (26).

In addition to the label-free off-gel profiling described above, we started a quantitative evaluation of the differences in protein abundance between the BEC with limited and reinduced BBB functions via an isotope-coded protein label profiling approach using a commercially available kit (Serva, Heidelberg, Germany). After their fractionation by increasing acetonitrile concentration, the proteins in each fraction (F0, F25, F50, F75) from cells with reinduced $\mathrm{BBB}$ functions were labelled with the heavy isotope. Then, each fraction from reinduced BBB was mixed with the equivalent one from the limited BBB cells 
that were labelled with the light isotope. The consecutive nano-LC MALDI-TOF/TOF-MS analyses were performed in triplicate. Among the 412 proteins that were identified in at least two of the three replicates, 290 were quantified and when a threshold of 1.3 was retained for the regulation factor, 81 proteins were shown to be upregulated in BEC, with 34 and 47 in reinduced BBB and in limited BBB, respectively (28).

The combination of in-gel and off-gel approaches enabled the identification of about 430 Triton X-100soluble proteins from BEC with reinduced BBB functionalities (27) and thus constituted a first proteome reference for those cells. Information on the proteins identified after 1D-LC MS/MS was shared through the PRIDE database for convenient comparison with proteomic datasets from non-brain vascular endothelial cells (http://www. ebi.ac.uk/pride project PRD000250, accession numbers 12825-12830). Due to the extraction conditions, only a few proteins $(<5 \%)$ were membrane-associated proteins. More than $75 \%$ of the identified proteins display binding, catalytic or structural functions. Most identified proteins were involved in metabolic and cellular processes but transport and cell-cell communication process accounted for almost $25 \%$ of the identified species. This also emphasized the advantage of interspecies sequence homology comparisons for protein identification in noncompletely sequenced genomes and highlighted the drifts and drawbacks generated by the rapid, gel-free proteomic methods which, nevertheless, provide most of today's data.

The distribution and nature of PM proteins in BEC was assessed after surface biotin labelling, isolation of the labelled proteins with streptavidin affinity chromatography and identification with nano-LC MS/MS. Very few cytoplasmic proteins, secreted proteins or proteins added to the cell culture medium were recovered-despite their relatively high cellular abundance. We reported (29) on the novel identification of transmembrane and membraneassociated proteins in bovine BEC with reinduced BBB phenotype. Our findings demonstrated the efficiency of the enrichment approach used, even though only about 30 proteins came from the BEC PM. The fact that transmembrane and membrane-associated proteins accounted for less than half the identified proteins showed how difficult it still is to isolate, solubilize and digest hydrophobic proteins of low cellular abundance. Our results suggest that the specific properties of PM proteins must be taken into account when seeking to improve biotinylation, purification and identification methods. Furthermore, this study reported the identification of several proteins involved in cellular endocytosis, membrane trafficking and receptor internalization (such as homology domain-containing protein 2 and myoferlin) together with their cellular partners. These proteins and the pathways of which they are a part may become new targets for increasing drug transport across the BBB.

\section{Quantitative Targeted Proteomics Strategy}

The absolute quantification of multiple membrane proteins in very complex samples such as mammalian tissues can be studied by LC-MS combined with in silico peptide selection criteria, using multiple reaction monitoring. By applying this focused proteomic to PM transporter proteins it will be possible, for the first time (30), to determine simultaneously the expression level of multiple membrane transporters such as P-glycoprotein (ABCB1), breast cancer resistance protein (ABCG2), and glucose transporter 1 among others and provide a quantitative atlas of membrane transporter proteins. Transporters and efflux pumps are numerous and the understanding of the regulation of the transport through the BBB could indicate alternative ways of reaching the brain. Therefore, this technique became a new area for pharmaceutical research, providing the quantification of known proteins localized at the membrane of the brain microvessels (18).

The protein expression of relevant transporters and other proteins in the $\mathrm{BEC}$ isolated from wild-type mice and ABCB1, ABCG2, and ABCB1/ABCG2 knockout mice was examined with a quantitative targeted proteomics approach (31). It was shown that there are no changes in the expression of several relevant transporters in BEC isolated from single and combination knockout mice. These data suggested that the mechanism behind the functional compensation between $\mathrm{ABCB} 1$ and $\mathrm{ABCG} 2$ at the $\mathrm{BBB}$ is not related to compensatory changes in transporter expression.

The previously presented studies revealed a large number of applications for proteomic analysis. Indeed, these methods provide information on cell behavior under physiological conditions or in cell cultures. Proteomics normally provide information about specific markers; unfortunately, in the case of BBB, it has not been possible to measure the $\gamma$-glutamyl-transpeptidase, monoaminoacid oxidase and alkaline phosphatase protein expression levels by proteomics, probably due to their relatively low abundance and their particular properties, which implies difficulties in solubilization and necessitate further treatment for proteomic research. These BBB markers were never detected on 2-DE gels and no data are available in relevant literature on their identification using liquid chromatography. Nevertheless, proteomics includes techniques for analyzing compounds, degradation products, receptors and transporters and opened a new route for understanding the BBB and brain targeting.

\section{Applications for BBB Diseases and Dysfunction}

BBB dysfunction in neurodegenerative diseases and stroke have often been studied in order to perform specific therapeutic targeting to avoid damage of the brain area concerned and also to design rapid diagnostic tools. Stroke is blood-flow failure in a particular brain area. To mimic 
435 stroke, various models have been used. In vivo models are
436 based on artificial obstruction of blood flow in the brain leading to a lack of nutrients and oxygen. The in vitro model involves cell oxygen deprivation named hypoxia, and oxygen and glucose deprivation (OGD or ischemia). The variation in protein expression during hypoxia, and post-hypoxic reoxygenation was initially studied using rat BEC and analyzed with 2-DE (32). The proteins with changes in expression levels could be sorted into three categories, proteins from mitochondria and endoplasmic reticulum, proteins associated with the cytoskeleton, and proteins of the glycolysis pathway. After hypoxia, protein expression and the enzymatic activity of the glycolysis pathway were upregulated. These results suggest that endothelial cells respond to the hypoxic stress with an increase in glucose metabolism. The modulation of cytoskeletonassociated proteins implies a cell structure rearrangement. Finally, during post-hypoxic reoxygenation, upregulation of most proteins was reduced towards control levels, indicating that, under the used conditions, hypoxia-induced metabolic overexpression is reversible (32).

The study of the BBB in vivo is the most efficient way of investigating a stroke response. The isotope coded affinity tag method (33) was used to demonstrate the variation in protein expression after $20 \mathrm{~min}$ of transient global cerebral ischemia, and 1, 6 and $24 \mathrm{~h}$ after reperfusion in rats. Laser capture dissection provided $\sim 300$ captured microvessels that permitted the identification of 50 proteins with a significant expression variation. Upregulation of the proteins in the early step $(1 \mathrm{~h})$ reveals a modification of the cells for an inflammatory and a proliferative phenotype with proteins like transcription factors and signal transduction molecules. Most returned to a basal level after $6 \mathrm{~h}$ of reperfusion. After $24 \mathrm{~h}$ of reperfusion, a second wave of upregulation appears with proteins like inflammatory cytokines and metalloproteases. These changes seem to be correlated with the BBB disruption observed in this pathology (33).

Comparison of 2-DE and isotope-coded affinity tag method methods showed their complementarities and helped completing our knowledge on ischemia/reperfusion (34). This study, carried out with rat brain endothelial cells immortalized by a stable transfection of SV40 large T antigen (35), presents $\sim 200$ identified proteins with a significant variation, sorted by their functions. On the one hand, glucose metabolism, stress proteins and antioxidative defense are involved in the endothelial response to brain ischemia; indeed, after reperfusion proteins from these groups were increased. This emphasizes activation of endothelial cell survival mechanisms. On the other hand, a new protein category appears with free radical detoxification proteins such as superoxide dismutase, formaldehyde dehydrogenase, and thioredoxin and metallothionein. Finally, the BEC display an altered polarization that could be involved in the changed permeability of the BBB during ischemia/reperfusion (34). In addition, the increase in heavy and light chains of clathrin indicates increased endocytosis. To conclude, brain hypoxia and ischemia studied by proteomic methods suggest, first, that glucose metabolism is upregulated early during an oxygen stress and, second, that BEC respond to the lack of oxygen with free radical detoxification proteins. Finally, these results also show a strong communication between the BEC and their environment and tissue modelling following inflammation.

The effects of edaravone- a free radical scavenger used for acute ischemic stroke on human BEC - was elucidated by 2D-DIGE (36). Thirty eight protein spots whose intensity was significantly altered by the edaravone treatment were found and among them 17 proteins were successfully identified. Four of those proteins were cytoskeleton proteins or cytoskeleton-regulating proteins. The edaravone-treated cells induced the merging of a tight junction protein, zona occludens-1, along the junction of the cells. In addition, edaravone suppressed interleukin-1 $\beta$-induced secretion of monocyte chemoattractant protein-1, which was reported to increase cell permeability. These data provided fundamental and useful information in the clinical use of edaravone in patients with cerebral vascular diseases on the one hand and on the other highlighted the relevance of clinical proteomics.

A recent study evaluated the benefits of statins when administered during the acute phase of stroke and explored its mechanisms of action through brain proteomics assay. Using an embolic model, simvastatin-treated rats showed significant infarct volume reduction and neurological improvement compared to vehicle-treated group. The analysis of brain homogenates by DIGE technology concluded that the protective effect of simvastatin can be attributable to oxidative stress response attenuation and BBB protection after cerebral ischemia (37).

Redox proteomics allows the identification of specific targets of protein oxidation in a biological sample. Using proteomic techniques, apolipoprotein A-I (ApoA-I) has been found at decreased levels in subjects with a variety of neurodegenerative disorders including in the serum and cerebrospinal fluid of Alzheimer disease, Parkinson disease, and Down syndrome with gout. ApoA-I plays roles in cholesterol transport and regulation of inflammation. Redox proteomics further showed ApoA-I to be highly oxidatively modified and particularly susceptible to modification by 4-hydroxy-2-trans-nonenal, a lipid peroxidation product. Current evidence suggests ApoA-I to be a promising diagnostic marker as well as a potential target for therapeutic strategies in these neurodegenerative disorders (38).

In an effort to understand the BBB damage and the transendothelial migration of HIV-infected leucocytes in the CNS, the effects of HIV-1-infected macrophages on human BEC protein profiles were determined using a differential proteomics approach (39). HIV-1 infected monocyte-derived macrophages induced the upregulation 
of $>200$ human BEC proteins. These included metabolic proteins, voltage-gated ion channels, heat shock, transport, cytoskeletal, regulatory, and calcium binding proteins. It was concluded that HIV-1-infected monocyte-derived macrophages affect the human BEC proteome and, in this way, contribute to BBB dysfunction and the development of HIV-1 CNS disease.

Downregulation of 47 proteins in the cerebral microvessels from the cerebral cortex of mice with diet-induced obesity was demonstrated using comparative proteomics. The protein data set included cytoskeletal proteins, chaperons, enzymes, transport-related proteins, and regulators for transcriptional and translational activities. Only two proteins involved in mRNA transport and processing were upregulated. The predominant downregulation suggested that diet-induced obesity suppresses metabolic activity of BBB microvessels. This finding may provide novel mechanistic insight into how obesity influences CNS function via regulatory changes of the BBB (40).

The pathomechanisms of multiple sclerosis were investigated in a 2-DE study by treating a human brain capillary endothelial cell transfected with a plasmid from SV40 cultured with serum of multiple sclerosis patients or with this serum and interferon- $\beta 1 \mathrm{~b}$ (41). Several 14-3-3 isoform proteins are involved in the response to multiple sclerosis at the BBB. Nevertheless, according to the isoform there is variation in regulation, for example, 14-3-3 epsilon increases with serum, whereas 14-3-3 zeta/delta decreases. Some proteins up- or downregulated with multiple sclerosis serum are also modulated by interferon- $\beta 1 \mathrm{~b}$ treatment. For example, annexin 1 decreases with serum treatment and this phenomenon is reversed with interferon- $\beta 1 b$ (41). Other proteins regulated by these treatments were identified such as Ras-related protein, plasminogen and ribonuclease/angiogenin inhibitor 1 . This shows that angiogenesis may play a role in the pathogenesis of multiple sclerosis. Lastly, interferon- $\beta 1 \mathrm{~b}$ induces the expression of $70-\mathrm{kDa}$ heatshock protein possibly linked to an immunomodulatory effect of the multiple sclerosis treatment (41).

A cellular toxicity analysis of compounds in cigarette smoke was carried out with rat BEC activated by nicotine and polyaromatic hydrocarbon. This study shows variations in protein abundance between Triton X-100-soluble and Triton X-100-insoluble fractions of tight junction proteins zona occludens- 1 , Occludin, Claudin-5 and catenin. It is known that Triton-X-100 partially solubilizes the membranes and the cortical cytoskeleton. In addition to these results, a 2-DE study was carried out, highlighting that heat-shock proteins are involved in this stress response. Proteins that link the cytoskeleton to the adhesion plaques of the membrane such as metavinculin and zyxin and proteins related to the translation were also identified (42). Toxic compounds found in cigarette smoke could induce brain capillary dysfunctions linked to the cytoskeleton organization, tight junction modifications and modulation of the cell-cell adhesion with proteins of the adhesion plaques. To conclude, these compounds appear as potentially dangerous molecules for brain homeostasis.

\section{Conclusions and Future Prospects}

The importance of quantitative proteomic analysis in BBB research is increasing and will lead to a better understanding of patients' BBB function as well as improving methodology for drug delivery to the brain. We expect quick developments after the publication of the draft of the human proteome (43). A specialized database, ProteomicsDB (https://www.proteomicsdb.org/), which is a joint effort of the Technische Universität München and SAP AG, is dedicated to expedite the identification of the human proteome and its use across the scientific community. This will rapidly improve the available proteomic information about the healthy human BBB. A recent review (19) emphasized the important need to investigate, in the future, the BBB of patients with CNS diseases. An extensive investigation of gene and protein patterns of transporters and metabolizing enzymes carried out on isolated brain microvessels and cortex biopsies from 12 patients with epilepsy or glioma provided molecular information for understanding drug entry and metabolism in the human BBB (44).

Indeed, obtaining human brain capillaries is a significant barrier to such analysis and the development of human in vitro BBB models will greatly help. Recently, it was shown that endothelial cells derived from human pluripotent stem cell lines acquire BBB properties when codifferentiated with neural cells that provide relevant cues, including those involved in $\mathrm{Wnt} / \beta$-catenin signaling (45). The resulting endothelial cells have many BBB attributes. They respond to co-culture with astrocytes and acquire substantial barrier properties. It may be possible to use patients' endothelial cells derived from their own pluripotent stem cells to evaluate BBB protein constituents (19). A human BBB model using cord blood-derived hematopoietic stem cells was recently generated (46). The model is reproducible because it can be generated from stem cells isolated from different donors and in different laboratories. The cells were initially differentiated into ECs followed by the induction of BBB properties by coculture with pericytes. Evidence was again provided that $\mathrm{Wnt} / \beta$-catenin signaling pathway mediates in part (46) the BBB inductive properties of pericytes (47).

As recently stated (19), to understand the human BBB, it will be necessary to identify expressed molecules, to clarify interspecies and in vivo-in vitro differences, and to estimate transport function in humans. Highly sensitive, accurate and specific protein quantification by quantitative targeted proteomics is an essential methodology for human BBB studies. By completing the current investigations, a thorough proteomic analysis will provide additional
604

605

606

607

608

609

610

611

612

613

614

615

616

617

618

619

620

621

622

623

624

625

626

627

628

629

630

631

632

633

634

635

636

637

638

639

640

641

642

643

644

645

646

647

648

649

650

651

652

653

654 
information concerning brain pathologies or BBB metabolism. Indeed, new questions will arise from proteomics data, which have to be answered by applying the complete life sciences methodology (11). New fields will emerge and will provide new challenges for protein expression profiling and other technologies in the future.

\section{Acknowledgments}

Q1 This research was funded by the Ministère de l'Education Nationale, de de l'Enseignement Supérieur et la Recherche. We wish to thank Drs. Barbara Deracinois, Sophie Duban-Deweer and Gwënaël Pottier, the main contributors of the proteomics project of the laboratory, Johan Hachani for technical expertise and continuous help and Dr. Rigas Karamanos for linguistic advice.

\section{References}

1. Cecchelli R, Berezowski V, Lundquist S, et al. Modelling of the bloodbrain barrier in drug discovery and development. Nat Rev Drug Discov 2007;6:650-661

2. Armulik A, Genové G, Mäe M, et al. Pericytes regulate the bloodbrain barrier. Nature 2010;468:557-561.

3. Abbott NJ, Patabendige AAK, Dolman DEM, et al. Structure and function of the blood-brain barrier. Neurobiol Dis 2010;37:13-25.

4. Nag S. Morphology and properties of brain endothelial cells. Methods Mol Biol 2011;686:3-47.

5. Hawkins BT, Davis TP. The blood-brain barrier/neurovascular unit in health and disease. Pharmacol Rev 2005;57:173-185.

6. Howes MT, Mayor S, Parton RG. Molecules, mechanisms, and cellular roles of clathrin-independent endocytosis. Curr Opin Cell Biol 2010;22:519-527.

7. Kelly BT, Owen DJ. Endocytic sorting of transmembrane protein cargo. Curr Opin Cell Biol 2011;23:404-412.

8. O'Bryan JP. Intersecting pathways in cell biology. Sci Signal 2010;3: re10.

9. Reider A, Wendland B. Endocytic adaptors-social networking at the plasma membrane. J Cell Sci 2011;124:1613-1622.

10. Leth-Larsen R, Lund RR, Ditzel HJ. Plasma membrane proteomics and its application in clinical cancer biomarker discovery. Mol Cell Proteomics 2010;9:1369-1382.

11. Pottiez G, Flahaut C, Cecchelli R, et al. Understanding the blood-brain barrier using gene and protein expression profiling technologies. Brain Res Rev 2009;62:83-98.

12. Calabria AR, Shusta EV. Blood-brain barrier genomics and proteomics: elucidating phenotype, identifying disease targets and enabling brain drug delivery. Drug Discov Today 2006;11:792-799.

13. Lu L, Yang PY, Rui YC, et al. Comparative proteome analysis of rat brain and coronary microvascular endothelial cells. Physiol Res 2007;56:159-168.

14. Pellieux C, Desgeorges A, Pigeon $\mathrm{CH}$, et al. Cap G, a gelsolin family protein modulating protective effects of unidirectional shear stress. $\mathrm{J}$ Biol Chem 2003;278:29136-29144.

15. Ohtsuki S, Uchida Y, Kubo Y, et al. Quantitative targeted absolute proteomics-based ADME research as a new path to drug discovery and development: methodology, advantages, strategy, and prospects. J Pharm Sci 2011;100:3547-3559.

16. Deracinois B, Flahaut C, Duban-Deweer S, et al. Comparative and quantitative global proteomics approaches: an overview. Proteomes 2013;1:180-218.

17. Shusta EV. Blood-brain barrier genomics, proteomics, and new transporter discovery. NeuroRx 2005;2:151-161.
18. Ohtsuki S, Terasaki T. Contribution of carrier-mediated transport systems to the blood-brain barrier as a supporting and protecting interface for the brain; importance for CNS drug discovery and development. Pharm Res 2007;24:1745-1758.

19. Ohtsuki S, Hirayama M, Ito S, et al. Quantitative targeted proteomics for understanding the blood-brain barrier: towards pharmacoproteomics. Expert Rev Proteomics 2014;11:303-313.

20. Murugesan N, Macdonald JA, Lu Q, et al. Analysis of mouse brain microvascular endothelium using laser capture microdissection coupled with proteomics. Methods Mol Biol 2011;686:297-311.

21. Pottiez G, Sevin E, Cecchelli R, et al. Actin, gelsolin and filamin-A are dynamic actors in the cytoskeleton remodelling contributing to the blood brain barrier phenotype. Proteomics 2009;9:1207-1219.

22. Dehouck MP, Jolliet-Riant P, Brée F, et al. Drug transfer across the blood-brain barrier: correlation between in vitro and in vivo models. J Neurochem 1992;58:1790-1797.

23. Duban-Deweer S, Flahaut C, Karamanos Y. The proteome of brain capillary endothelial cells: towards a molecular characterization of an in vitro blood-brain barrier model. In: Karamanos Y, ed. Expression Profiling in Neuroscience. New York: Humana Press; 2012. pp. 161-179.

24. Pottiez G, Duban-Deweer S, Deracinois B, et al. A differential proteomic approach identifies structural and functional components that contribute to the differentiation of brain capillary endothelial cells. J Proteomics 2011;75:628-641.

25. Deracinois B, Pottiez G, Chafey P, et al. Glial-cell-mediated reinduction of the blood-brain barrier phenotype in brain capillary endothelial cells: a differential gel electrophoresis study. Proteomics 2013; 13:1185-1199.

26. Deracinois B, Duban-Deweer S, Pottiez G, et al. TNAP and EHD1 are over-expressed in bovine brain capillary endothelial cells after the re-induction of blood-brain barrier properties. PLoS One 2012; 7:e48428.

27. Pottiez G, Deracinois B, Duban-Deweer S, et al. A large-scale electrophoresis- and chromatography-based determination of gene expression profiles in bovine brain capillary endothelial cells after the re-induction of blood-brain barrier properties. Proteome Sci 2010;8:57.

28. Karamanos Y. Studying molecular aspects of the blood-brain barrier using an in vitro model: contribution of a global proteomics strategy. J Biosci Med 2014;02:18-25.

29. Duban-Deweer S, Hachani J, Deracinois B, et al. Proteomic analysis of plasma membrane proteins in an in vitro blood-brain barrier model. In: Man TK, Flores J, eds. Proteomics-Human Diseases and Protein Functions. Rijeka, Croatia: InTech; 2012. pp. 391-416.

30. Kamiie J, Ohtsuki S, Iwase R, et al. Quantitative atlas of membrane transporter proteins: development and application of a highly sensitive simultaneous LC/MS/MS method combined with novel in-silico peptide selection criteria. Pharm Res 2008;25:1469-1483.

31. Agarwal S, Uchida Y, Mittapalli RK, et al. Quantitative proteomics of transporter expression in brain capillary endothelial cells isolated from P-glycoprotein (P-gp), breast cancer resistance protein (Bcrp), and P-gp/Bcrp knockout mice. Drug Metab Dispos 2012;40: 1164-1169.

32. Haseloff RF, Krause E, Bigl M, et al. Differential protein expression in brain capillary endothelial cells induced by hypoxia and posthypoxic reoxygenation. Proteomics 2006;6:1803-1809.

33. Haqqani AS, Nesic M, Preston E, et al. Characterization of vascular protein expression patterns in cerebral ischemia/reperfusion using laser capture microdissection and ICAT-nanoLC-MS/MS. FASEB J 2005;19:1809-1821.

34. Haqqani AS, Kelly J, Baumann E, et al. Protein markers of ischemic insult in brain endothelial cells identified using 2D gel electrophoresis and ICAT-based quantitative proteomics. J Proteome Res 2007;6: 226-239. 
35. Zhang W, Mojsilovic-Petrovic J, Andrade MF, et al. The expression and functional characterization of ABCG2 in brain endothelial cells and vessels. FASEB J 2003;17:2085-2087.

36. Onodera H, Arito M, Sato T, et al. Novel effects of edaravone on human brain microvascular endothelial cells revealed by a proteomic approach. Brain Res 2013;1534:87-94.

37. Campos-Martorell M, Salvador N, Monge M, et al. Brain proteomics identifies potential simvastatin targets in acute phase of stroke in a rat embolic model. J Neurochem 2014;130: 301-312.

38. Keeney JTR, Swomley AM, Förster S, et al. Apolipoprotein A-I: insights from redox proteomics for its role in neurodegeneration. Proteomics Clin Appl 2013;7:109-122.

39. Ricardo-Dukelow M, Kadiu I, Rozek W, et al. HIV-1 infected monocyte-derived macrophages affect the human brain microvascular endothelial cell proteome: new insights into blood-brain barrier dysfunction for HIV-1-associated dementia. J Neuroimmunol 2007; 185:37-46.

40. Ouyang S, Hsuchou H, Kastin AJ, et al. Diet-induced obesity suppresses expression of many proteins at the blood-brain barrier. J Cereb Blood Flow Metab 2014;34:43-51.
41. Alexander JS, Minagar A, Harper M, et al. Proteomic analysis of human cerebral endothelial cells activated by multiple sclerosis serum and IFNbeta-1b. J Mol Neurosci 2007;32:169-178.

42. Hutamekalin P, Farkas AE, Orbók A, et al. Effect of nicotine and polyaromatic hydrocarbons on cerebral endothelial cells. Cell Biol Int 2008;32:198-209.

43. Wilhelm M, Schlegl J, Hahne H, et al. Mass-spectrometry-based draft of the human proteome. Nature 2014;509:582-587.

44. Shawahna R, Uchida Y, Declèves X, et al. Transcriptomic and quantitative proteomic analysis of transporters and drug metabolizing enzymes in freshly isolated human brain microvessels. Mol Pharmacol 2011;8:1332-1341

45. Lippmann ES, Azarin SM, Kay JE, et al. Derivation of blood-brain barrier endothelial cells from human pluripotent stem cells. Nat Biotechnol 2012;30:783-791.

46. Cecchelli R, Aday S, Sevin E, et al. A stable and reproducible human blood-brain barrier model derived from hematopoietic stem cells. PLoS One 2014;9:e99733.

47. Nakagawa S, Deli MA, Kawaguchi H, et al. A new blood-brain barrier model using primary rat brain endothelial cells, pericytes and astrocytes. Neurochem Int 2009;54:253-256. 